\section{(1)}

CrossMark

\title{
Health-related quality of life and risk factors associated with spirometric restriction
}

\author{
Stefano Guerra ${ }^{1,2,3,4}$, Anne-Elie Carsin ${ }^{2,3,4,5}$, Dirk Keidel (10) 6,7 \\ Jordi Sunyer ${ }^{2,3,4,5}$, Bénédicte Leynaert ${ }^{8,9}$, Christer Janson ${ }^{10}$, Debbie Jarvis ${ }^{11}$, \\ Daiana Stolz ${ }^{12}$, Thomas Rothe ${ }^{13}$, Marco Pons ${ }^{14}$, Alexander Turk ${ }^{15}$, \\ Josep M. Anto $2,3,4,5$ and Nicole Probst-Hensch 6,7
}

\begin{abstract}
Affiliations: 'Asthma and Airway Disease Research Center, University of Arizona, Tucson, AZ, USA. ${ }^{2}$ ISGlobal, Center for Research in Environmental Epidemiology (CREAL), Barcelona, Spain. ${ }^{3}$ Universitat Pompeu Fabra, Barcelona, Spain. ${ }^{4}$ CIBER Epidemiología y Salud Pública (CIBERESP), Spain. ${ }^{5}$ IMIM (Hospital del Mar Medical Research Institute), Barcelona, Spain. 'Swiss Tropical and Public Health Institute, Basel, Switzerland. ${ }^{7}$ University of Basel, Basel, Switzerland. ${ }^{8}$ Inserm, UMR 1152, Pathophysiology and Epidemiology of Respiratory Diseases, Epidemiology Team, Paris, France. ${ }^{9}$ University Paris Diderot Paris 7, UMR 1152, Paris, France. ${ }^{10}$ Respiratory, Allergy and Sleep Research, Dept of Medical Sciences, Uppsala University, Uppsala, Sweden. ${ }^{11}$ National Heart and Lung Institute, Imperial College London, London, UK. ${ }^{12}$ Clinic of Pulmonary Medicine and Respiratory Cell Research, University Hospital Basel, Basel, Switzerland. ${ }^{13}$ Zürcher RehaZentren, Davos Platz, Switzerland. ${ }^{14}$ Ospedale Regionale di Lugano - Sede Civico, Lugano, Switzerland. ${ }^{15}$ Zürcher RehaZentren, Faltigberg-Wald, Switzerland.
\end{abstract}

Correspondence: Stefano Guerra, Asthma and Airway Disease Research Center, University of Arizona, Tucson, AZ 85724, USA. E-mail: stefanodemail.arizona.edu

@ERSpublications

Restrictive spirometric pattern is linked to worse health-related QoL, partly independent of respiratory symptoms http://ow.ly/RTU33096m8i

Cite this article as: Guerra S, Carsin A-E, Keidel D, et al. Health-related quality of life and risk factors associated with spirometric restriction. Eur Respir J 2017; 49: 1602096 [https://doi.org/10.1183/ 13993003.02096-2016].

ABSTRACT The restrictive spirometric pattern is associated with a substantial morbidity and mortality burden. We sought to determine to what extent spirometric restriction is associated with impaired quality of life.

We used data from two large population-based European cohorts: 6698 European Community Respiratory Health Survey (ECRHS) and 6069 Swiss Cohort Study on Air Pollution and Lung and Heart Diseases in Adults (SAPALDIA) adult participants. The restrictive pattern was defined as forced expiratory volume in $1 \mathrm{~s}$ $(\mathrm{FEV} 1) /$ forced vital capacity $(\mathrm{FVC}) \geqslant$ lower limit of normal (LLN) and FVC $<\mathrm{LLN}$; an obstructive pattern was defined as FEV1/FVC <LLN independent of FVC. The Physical Component Summary and Mental Component Summary of quality of life were computed using the Short Form-36 questionnaire.

In both cohorts, the restrictive pattern was associated with heavy smoking, being underweight or obese and the coexistence of respiratory symptoms. In univariate analyses, compared with the normal group, both the restrictive and obstructive pattern had significant Physical Component Summary deficits $(-2.77$ and -2.08 , respectively, in ECRHS; -3.25 and -2.14 , respectively, in SAPALDIA; all p-values $<0.001)$. However, in models adjusted for sex, age, education, body mass index, smoking, comorbidities and respiratory symptoms, only the restrictive pattern remained significantly associated with Physical Component Summary deficits ( $\mathrm{p}=0.004$ in ECRHS; $\mathrm{p}=0.001$ in SAPALDIA).

The restrictive spirometric pattern is associated with deficits in the physical component of quality of life that are partly independent of the presence of respiratory symptoms. 


\section{Introduction}

In recent years multiple population-based studies have shown that a significant proportion of the general adult population has a restrictive pattern on spirometry, characterised by reduced levels of forced expiratory volume in $1 \mathrm{~s}(\mathrm{FEV} 1)$ and forced vital capacity (FVC), and normal FEV1/FVC ratio [1-4]. Although the determinants and biological bases of this restrictive pattern remain largely unknown, subjects with spirometric restriction have been shown to be more likely to have cough and phlegm, shortness of breath with wheezing and dyspnoea [3,5-7], and suffer from and die of a number of comorbidities, including coronary heart disease, hypertension, metabolic syndrome and diabetes [2, 8-12].

Owing to its high prevalence in the general population, the influence of spirometric restriction on health status is a relevant question. Several studies have found a significant association of the restrictive spirometric pattern with impaired health-related quality of life. Participants in the US National Health and Nutrition Examination Survey (NHANES) with spirometric restriction were more likely to report functional impairment and fair/poor health than were participants with no lung disease and normal lung function [3,7], and the magnitude of these effects was largely comparable to that seen in subjects with moderate chronic obstructive pulmonary disease (COPD). Similarly, spirometric restriction was found to be associated with worse respiratory-specific health status, as assessed by the St George's Respiratory Questionnaire, in both the COPDGene [5] and Spanish EPI-SCAN [4] studies. However, to what extent the decreased health-related quality of life in spirometric restriction is dependent on the coexistence of respiratory symptoms and comorbidities remains to be determined.

The goal of our study was to determine whether the restrictive spirometric pattern is associated with impaired quality of life, independent of other known predictors, in two large population-based European cohorts of adults.

\section{Materials and methods}

\section{Study design and populations}

For the current study, we used data from the adult cohorts of the European Community Respiratory Health Survey (ECRHS) and the Swiss Cohort Study on Air Pollution and Lung and Heart Diseases in Adults (SAPALDIA) to perform a cross-sectional analysis in which spirometric patterns and quality of life were assessed at the same follow-up survey.

ECRHS is a multicentre study involving mainly centres in Europe. Detailed methods and full protocol of the study are available at www.ecrhs.org. Briefly, in 1991-1993, a random sample of individuals aged 20-44 years was enrolled. Participants completed a detailed questionnaire and spirometric lung function tests at baseline (ECRHS 1) and in a follow-up survey (ECRHS 2) taken $\sim 9$ years later. For the present study, we excluded from ECRHS analyses participants from the Basel centre who were enrolled in both the ECRHS and SAPALDIA studies. Although the ECRHS population was also enriched with a sample of subjects who reported taking asthma medication or having respiratory symptoms, for the present study we only used data from the random sample, with a total 6698 participants who completed a questionnaire and lung function tests at the follow-up survey. Among them, 5665 subjects (85\%) also completed the quality of life questionnaire (see the following section).

SAPALDIA is a study designed to investigate the effects of chronic exposure to ambient air pollution on respiratory and cardiovascular health in Switzerland, with protocols very similar to those implemented in ECRHS. In 1991, 9651 participants aged 18-60 years from eight areas representing the geographical diversity of Switzerland and covering three language regions were recruited for a detailed interview and health examination (SAPALDIA 1). The follow-up (SAPALDIA 2) was conducted in 2002, at which point 8047 participants provided health information. For the present study, we included a total of 6069 participants who completed questionnaire and lung function tests at the follow-up survey. Among them, 4906 subjects (81\%) also completed the quality of life questionnaire.

\section{Quality of life questionnaire}

The Short Form-36 (SF-36) questionnaire was administered to participants in the follow-up survey in both ECRHS and SAPALDIA. The SF-36 is a standardised, widely used and validated general health-related quality of life questionnaire [13]. It includes 36 questions from which the Physical Component Summary

Support statement: This study was supported by FIS award PS09/01354 from the Spanish Instituto de Salud Carlos III and grant 33CSCO-134276 from the Swiss National Science Foundation. Please refer to the supplementary material for a complete list of acknowledgements and funding for ECRHS 1 and 2. Funding information for this article has been deposited with the Crossref Funder Registry.

Conflict of interest: Disclosures can be found alongside this article at erj.ersjournals.com 
(PCS) and the Mental Component Summary (MCS) were computed as described in the SF-36 User's Manual [14]. By construction, in a general population the PCS and MCS scores have expected means of 50 and standard deviations of 10 [15]. A higher score indicates better quality of life.

\section{Covariates}

Smoking was categorised as never-smokers, ex-smokers and current smokers, where current smokers reported having smoked during the last month. Asthma was defined as a positive answer to the questions "Have you ever had asthma?" and "Was this confirmed by a doctor?". Consistent with previous reports [16], asthma onset was categorised as $\leqslant 10$ or $>10$ years of age. Chronic cough and phlegm were defined as a positive report of cough and phlegm that lasted for at least 3 months per year. Information on comorbidities was obtained through a questionnaire. All comorbidities that had available information in both ECRHS and SAPALDIA and a prevalence $\geqslant 0.5 \%$ were tested for association with spirometric patterns. Specific IgE levels were measured with the Pharmacia CAP System (Pharmacia Diagnostics, Uppsala, Sweden) for four allergens in ECRHS and with the Phadiatop test (Phadia, Uppsala, Sweden) for 11 allergens in SAPALDIA. Atopy was defined as having $>0.35 \mathrm{kU} \cdot \mathrm{L}^{-1}$ for any of the tested allergens.

\section{Spirometry}

In both ECRHS and SAPALDIA, pre-bronchodilator lung function was measured by spirometry. In order to identify subjects with low lung function in both populations using comparable methods, predictive equations for the fifth percentiles of FVC and FEV1/FVC were derived in never-smoking subjects without asthma using quantile regression. These fifth percentiles will be referred to as lower limit of normal (LLN). Both baseline and follow-up lung function values were included in the models, and sex-specific equations including age and height were determined.

A normal spirometric pattern was defined as FEV1/FVC $\geqslant L L N$ and FVC $\geqslant L L N$, a restrictive pattern as FEV1/ FVC $\geqslant$ LLN and FVC <LLN and an obstructive pattern as FEV1/FVC <LLN independent of FVC values. In both ECRHS and SAPALDIA, in main analyses the spirometric patterns were defined at the follow-up survey when quality of life was also measured. Spirometric patterns were also studied longitudinally based on the nine possible combinations of normal, restrictive and obstructive patterns at baseline and follow-up.

In sensitivity analyses the spirometric patterns based on fixed cut-off values were used, where the normal spirometric pattern was defined as $\mathrm{FEV} 1 / \mathrm{FVC} \geqslant 70 \%$ and $\mathrm{FVC} \geqslant 80 \%$ predicted, the restrictive pattern as $\mathrm{FEV} 1 / \mathrm{FVC} \geqslant 70 \%$ and $\mathrm{FVC}<80 \%$ predicted and the obstructive pattern as $\mathrm{FEV} 1 / \mathrm{FVC}<70 \%$ independent of FVC values. For these definitions, reference equations by HANKINSON et al. [17] were used.

\section{Statistical analyses}

Factors associated with spirometric patterns defined as a trichotomous outcome (normal/restrictive/ obstructive) were investigated separately in ECRHS and SAPALDIA using Chi-squared tests in univariate analyses and multinomial logistic regression adjusting variance estimates for clustering of data within centres in multivariate analyses. All factors that were associated with spirometric patterns at $\mathrm{p}<0.1$ in the univariate analysis were tested for inclusion in multivariate models.

Linear mixed models were used to assess the relationship between spirometric patterns and quality of life (PCS and MCS scores). In both the ECRHS and SAPALDIA analyses, centre was included as a random effect in the models to account for the correlation of residuals within the same area. To assess the influence of respiratory symptoms and comorbidity on the association between the spirometric patterns and health-related quality of life, a set of sequentially adjusted models was tested: the initial model (M0) was only adjusted for age, sex and education, while the subsequent models (M1-M4) were progressively adjusted for additional factors (i.e. body mass index (BMI), smoking, comorbidities and respiratory symptoms/asthma). Models were also tested after restricting analyses to subjects without respiratory symptoms (defined as the presence of wheezing, chronic bronchitis, dyspnoea or asthma). The same approach was used to investigate the association of longitudinal spirometry patterns with quality of life. In adjusted models, comorbidities were tested as both individual binary variables and as an overall comorbidity score, which facilitates testing for interactions. The score was obtained by regressing PCS (or MCS) values against all comorbidities in the same model and saving the predicted PCS (or MCS) score for each participant. As a result of previous reports of increased cardiovascular morbidity in subjects with spirometric restriction, interactions between the spirometric pattern and the presence of heart disease, hypertension or diabetes were also tested in models predicting PCS. All analyses were performed in Stata (StataCorp, College Station, TX, USA).

\section{Results}

In ECRHS, complete data on lung function, age, sex and height were available for 6698 out of the 8233 participants (81\%) (figure 1a). Compared with participants who were excluded from this study, those 

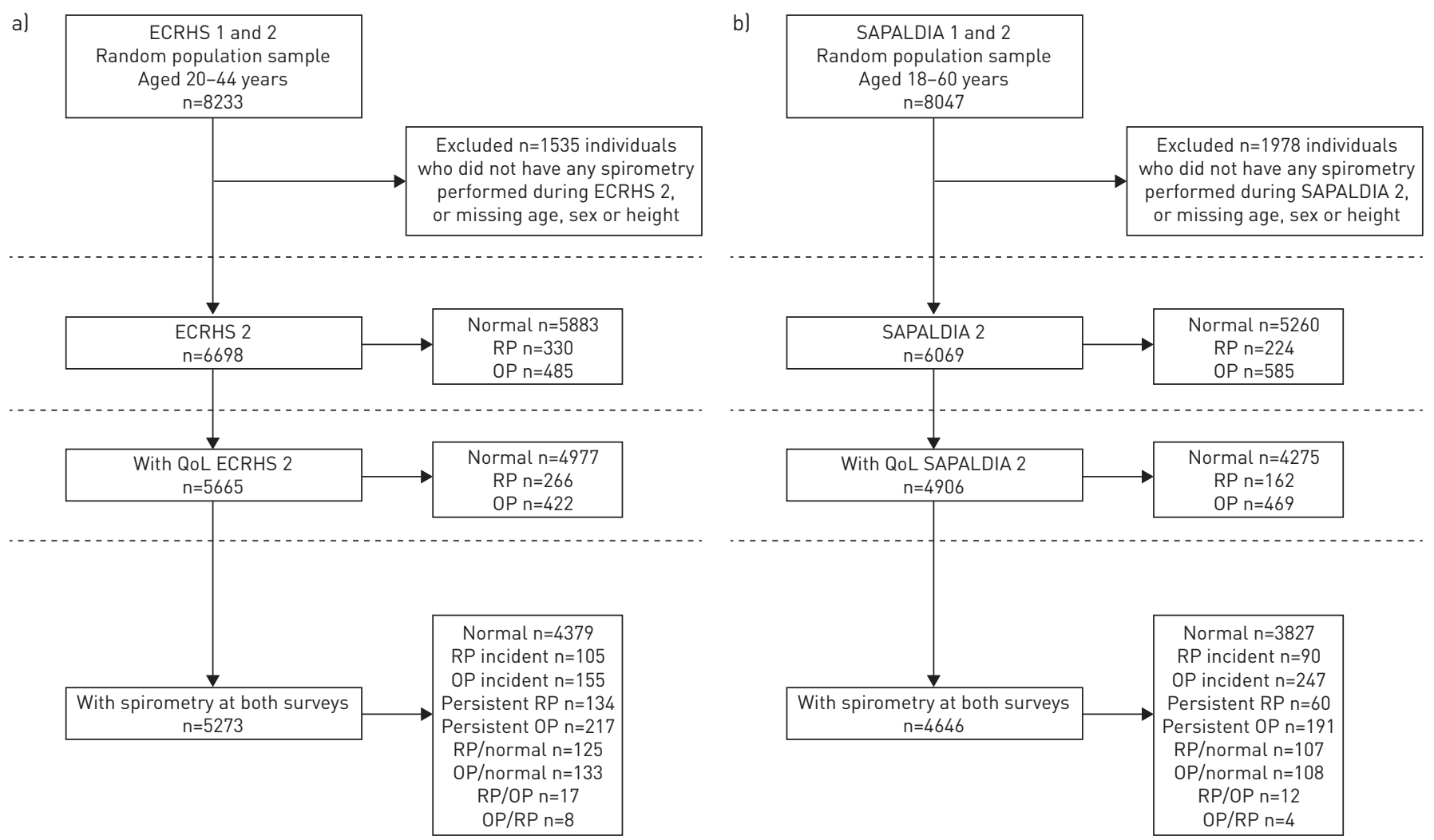

FIGURE 1 Sample selection for a) European Community Respiratory Health Survey (ECRHS) and b) Swiss Cohort Study on Air Pollution and Lung and Heart Diseases in Adults (SAPALDIA). RP: restrictive pattern; OP: obstructive pattern; QoL: quality of life.

included were younger and more likely to be males. No differences in smoking patterns were observed between included and excluded subjects. In SAPALDIA, complete data on lung function, age, sex and height were available for 6069 out of the 8047 participants (75\%) (figure 1b). Compared with participants who were excluded from this study, those included were younger and less likely to be current smokers, but no significant differences were observed in sex distribution. In ECRHS, among the 6698 subjects included in the analyses, $330(4.9 \%)$ had a restrictive pattern and $485(7.2 \%)$ had an obstructive pattern. In SAPALDIA, among the 6069 individuals included in analyses, 224 (3.7\%) had a restrictive pattern and 585 (9.6\%) had an obstructive pattern. When using the $80 \%$ cut-off in FVC \% predicted values to define restriction and the fixed $70 \%$ cut-off in the FEV1/FVC ratio to define obstruction, the corresponding frequencies were $6.6 \%$ for the restrictive pattern and $5.4 \%$ for the obstructive pattern in ECRHS, and $2.4 \%$ and $21.8 \%$, respectively, in SAPALDIA.

\section{Factors associated with spirometry patterns}

Table 1 shows the characteristics of subjects by spirometric patterns at ECRHS 2 and SAPALDIA 2. Distribution by smoking, BMI categories, parental asthma, physician-confirmed asthma and respiratory symptoms differed significantly across the spirometric patterns in both cohorts. Compared with normal subjects, participants in the obstructive but not the restrictive group were more likely to be ever-smokers. However, heavy smokers were more frequent in both the obstructive and the restrictive group. Increased BMI was only associated with the restrictive pattern, although in both cohorts $<25 \%$ of subjects in the restrictive group were obese. Whereas an asthma diagnosis was more frequent in both the restrictive and obstructive groups compared with normal subjects, parental asthma was only associated with the obstructive pattern. In ECRHS, but not SAPALDIA, the restrictive pattern was associated with a report of hospitalisation for lung disease in the first 2 years of life. Respiratory symptoms were most frequent in the obstructive group, with the restrictive group showing rates that were between those of normal subjects and obstructed subjects. The frequency of any comorbidity tended to be the highest in the restrictive group, although these associations were not significant. There were differences in the specific conditions associated with spirometric patterns in ECRHS and SAPALDIA (supplementary table E1), but the proportion of subjects who reported heart disease, hypertension or diabetes was higher in the restrictive group compared with the other two groups in both ECRHS $(\mathrm{p}=0.003)$ and SAPALDIA $(\mathrm{p}=0.066)$. 
In multivariate models (table 2), current and heavy smokers were more likely to have an obstructive pattern in both ECRHS and SAPALDIA, whereas associations between smoking and the restrictive pattern were inconsistent. In terms of BMI categories, the restrictive pattern was associated with being underweight in both

TABLE 1 Characteristics of individuals in the follow-up of the European Community Respiratory Health Survey (ECRHS 2) and Swiss Cohort Study on Air Pollution and Lung and Heart Diseases in Adults (SAPALDIA 2) by spirometry patterns ${ }^{\#}$

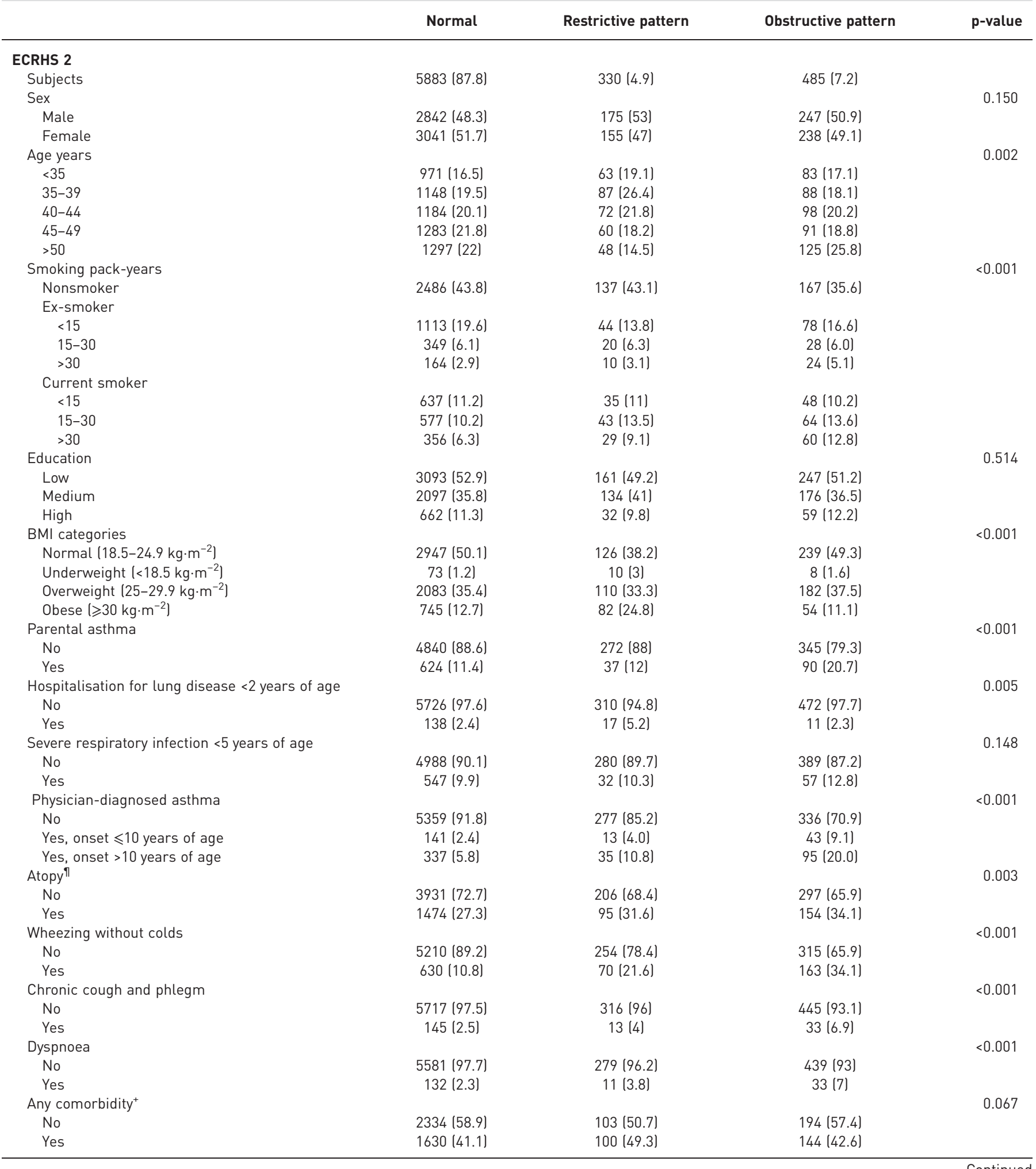




\section{Normal}

Restrictive pattern

Male

Female

Age years

$<40$

$40-44$

45-49

$50-54$

$55-59$

$\geqslant 60$

Smoking pack-years

Nonsmoker

Ex-smoker

$<15$

$15-30$

$>30$

Current smoker

$<15$

$15-30$

$>30$

Education

Low

Medium

High

BMI categories

Normal $\left(18.5-24.9 \mathrm{~kg} \cdot \mathrm{m}^{-2}\right)$

Underweight $\left(<18.5 \mathrm{~kg} \cdot \mathrm{m}^{-2}\right)$

Overweight $\left(25-29.9 \mathrm{~kg} \cdot \mathrm{m}^{-2}\right)$

Obese ( $\geqslant 30 \mathrm{~kg} \cdot \mathrm{m}^{-2}$ )

Parental asthma

No

Yes

Hospitalisation for lung disease $<2$ years of age

No

Yes

Severe respiratory infection $<5$ years of age

No

Yes

Physician-diagnosed asthma

No

Yes, onset $\leqslant 10$ years of age

Yes, onset $>10$ years of age

Atopy $^{\S}$

No

Yes

Wheezing without colds

No

Yes

Chronic cough and phlegm

No

Yes

Dyspnoea

No

Yes

Any comorbidity ${ }^{+}$

No

Yes
5260 (86.7)

$2511(47.7)$

$2749(52.3)$

224 (3.7)

$126(56.3)$

98 (43.8)

37 (16.5)

$23(10.3)$

34 (15.2)

34 (15.2)

37 (16.5)

59 (26.3)

97 (47.3)

25 (12.2)

8 (3.9)

$21(10.2)$

$10(4.9)$

15 (7.3)

29 (14.1)

15 (6.7)

$158(70.5)$

51 (22.8)

$91(40.6)$

$7(3.1)$

73 (32.6)

53 (23.7)

$202(90.2)$

22 (9.8)

214 (97.7)

$5(2.3)$

85 (1.6)

4831 (91.9)

426 (8.1)

4929 (93.9)

$118(2.2)$

200 (3.8)

$3249(69.6)$

$1420(30.4)$

4911 (93.4)

$345(6.6)$

4990 (98.2)

92 (1.8)

5051 (96.9)

162 (3.1)

2219 (42.3)

3029 (57.7)

207 (92.4)

$17(7.6)$

205 (92.3)

$4(1.8)$

13 (5.9)

$136(71.2)$

55 (28.8)

198 (88.4)

26 (11.6)

209 (95.9)

9 (4.1)

207 (92.8)

16 (7.2)

87 (38.8)

$137(61.2)$

\section{Obstructive pattern}

p-value

585 (9.6)
$278(47.5)$

307 (52.5)

86 (14.7)

$69(11.8)$

86 (14.7)

$85(14.5)$

77 (13.2)

182 (31.1)

194 (35.8)

71 (13.1)

35 (6.5)

53 (9.8)

30 (5.5)

37 (6.8)

122 (22.5)

49 (8.4)

$396(67.8)$

139 (23.8)

258 (44.2)

9 (1.5)

218 (37.3)

99 (17)

501 (85.6)

84 (14.4)

558 (97.7)

13 (2.3)

531 (90.8)

54 (9.2)

$462(79.7)$

32 (5.5)

86 (14.8)

348 (67.8)

165 (32.2)

446 (76.2)

139 (23.8)

512 (93.6)

$35(6.4)$

532 (92.4)

44 (7.6)

0.043

0.273

$<0.001$

0.002

0.003

0.001

0.444

0.606

$<0.001$

0.623

$<0.001$

$<0.001$

$<0.001$

229 (39.1)

356 (60.9)

Data are presented as $\mathrm{n}(\%)$, unless otherwise stated. BMI: body mass index. ${ }^{\#}$ : spirometric patterns defined based on lower limit of normal values (see Materials and methods); ${ }^{9}:>0.35 \mathrm{kU} \cdot \mathrm{L}^{-1}$ for any of the following allergens: Dermatophagoides pteronyssinus, timothy grass (Phleum pratense), cat and Cladosporium herbarum; ${ }^{+}$: see supplementary material for complete list of tested comorbidities; ${ }^{\S}:>0.35 \mathrm{kU} \cdot \mathrm{L}^{-1}$ for any of the following allergens: Cladosporium, D. pteronyssinus, Dermatophagoides farinae, cat, dog, horse, birch, timothy grass (P. pratense), mugwort (Artemisia), olive and Parietaria judaica (spreading pellitory). 
TABLE 2 Factors associated with spirometric patterns: relative risk ratios (95\% CI) for the restrictive and obstructive patterns in the follow-up of the European Community Respiratory Health Survey (ECRHS 2) and Swiss Cohort Study on Air Pollution and Lung and Heart Diseases in Adults (SAPALDIA 2) from multinomial logistic regressions

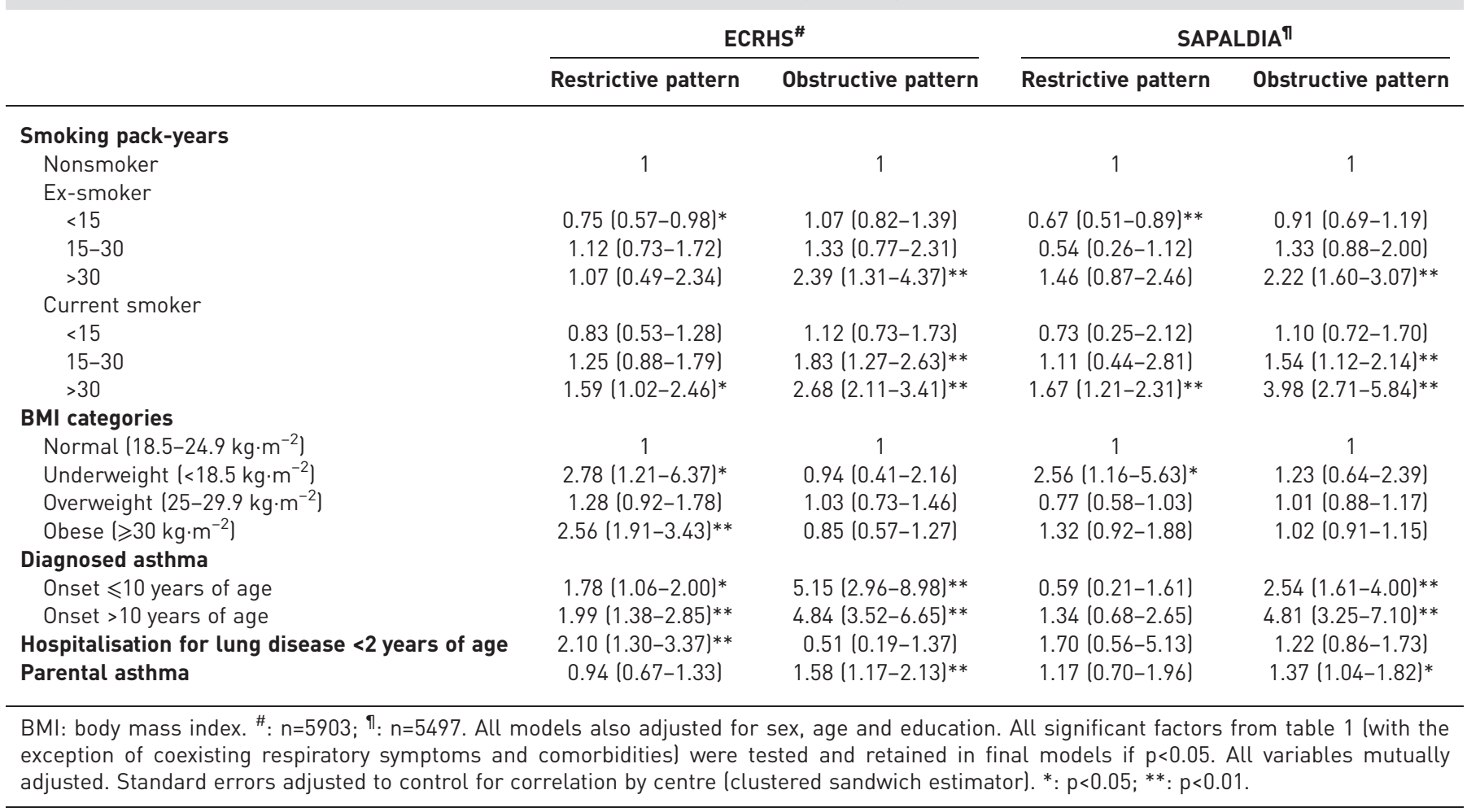

cohorts and with being obese only in ECRHS. Both a history of parental and personal asthma were associated with the obstructive pattern, whereas a history of hospitalisation for lung disease $<2$ years of age was a risk factor for the restrictive pattern, although this association reached statistical significance only in ECRHS.

Spirometric patterns and quality of life

Spirometry patterns were strongly associated with the PCS of quality of life in both cohorts (figure 2). Both the restrictive and the obstructive patterns had significantly lower PCS values than did the normal group ( $\mathrm{p}<0.001$ for both cohorts). In contrast, no significant differences of the MCS were found across spirometric patterns.

In ECRHS, compared with the normal group, the restrictive pattern had a 2.77 mean deficit $(\mathrm{p}<0.001)$ and the obstructive pattern had a 2.08 mean deficit $(\mathrm{p}<0.001)$ in PCS values. The corresponding mean deficits in SAPALDIA were $3.25(\mathrm{p}<0.001)$ and $2.14(\mathrm{p}<0.001)$, respectively. In multivariate models (table 3), the effects of the spirometric patterns on PCS values decreased in magnitude as they were progressively adjusted for multiple covariates. However, in the final fully adjusted models the restrictive pattern, unlike the obstructive pattern, remained significantly associated with PCS deficits compared with the normal group. Further adjustment for hospitalisations for lung disease $<2$ years of age did not cause any relevant change to the results.

In these sequential models, the largest reduction in coefficients for the obstructive pattern was observed after adjustment for respiratory symptoms in both ECRHS and SAPALDIA. In contrast, adjustment for respiratory symptoms had a smaller impact on the coefficients associated with the restrictive pattern. Results of the final model were very similar when comorbidities were included as individual binary variables or as an overall comorbidity score. A significant interaction of heart disease, hypertension and diabetes comorbidities with the restrictive spirometric pattern was observed in SAPALDIA ( $p$ for interaction=0.007), with the association between spirometric restriction and PCS deficits being stronger among participants with any of those comorbidities $(-6.07, \mathrm{p}<0.001)$ compared with participants with none of those comorbidities $(-1.61, \mathrm{p}=0.029)$. However, these results should be interpreted with caution because we did not find significant evidence for such an interaction in ECRHS. We also did not find consistent interactions between spirometric patterns and age across the two cohorts. 

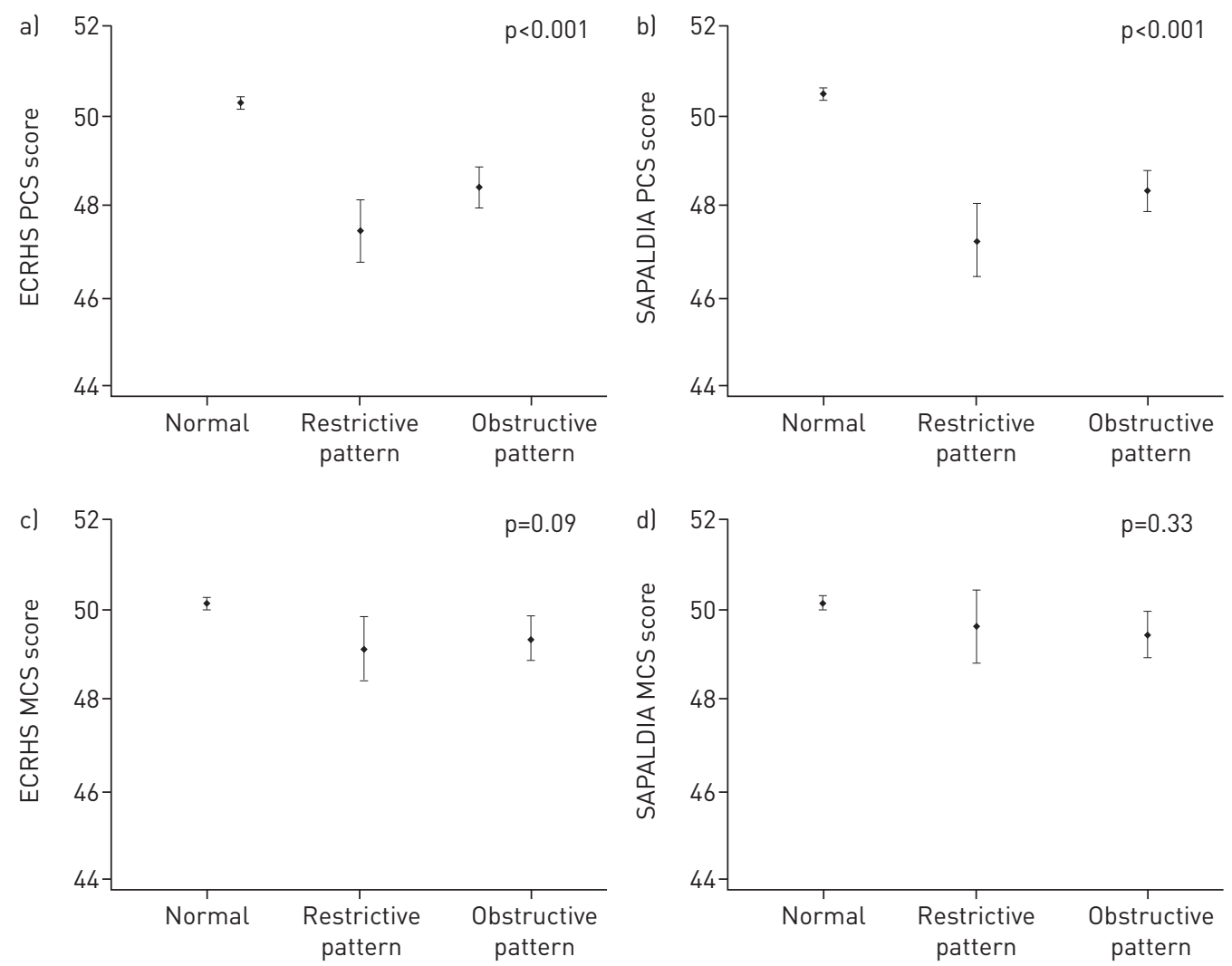

FIGURE 2 a, b) Physical Component Summary (PCS) and c, d) Mental Component Summary (MCS) by spirometric patterns for a, c) European Community Respiratory Health Survey (ECRHS) and b, d) Swiss Cohort Study on Air Pollution and Lung and Heart Diseases in Adults (SAPALDIA). Data are presented as mean \pm SE. p-values from ANOVA test.

When we restricted the adjusted M3 models from table 3 to subjects without respiratory symptoms ( $n=3244$ in ECRHS; $n=3657$ in SAPALDIA), we found again that the restrictive, but not the obstructive, group had significant PCS deficits compared with normal subjects $(-1.83, p=0.015$ and $-0.38, p=0.565$, respectively, in ECRHS; $-1.51, \mathrm{p}=0.041$ and $-0.51, \mathrm{p}=0.295$, respectively, in SAPALDIA).

The association of the restrictive pattern with PCS deficits was also confirmed in analyses using the fixed $70 \%$ cut-off in the FEV1/FVC ratio to define obstruction and the $80 \%$ cut-off in predicted FVC values to define restriction (supplementary table E2), and in analyses using the longitudinal spirometric patterns generated from the combination of cross-sectional spirometric patterns at the baseline and follow-up surveys (supplementary table E3). In the latter, in fully adjusted models subjects with a restrictive spirometric pattern in both surveys were the only group with a significant deficit of PCS values in both ECRHS and SAPALDIA ( $-2.37, \mathrm{p}=0.006$ in ECRHS; $-2.73, \mathrm{p}=0.009$ in SAPALDIA).

\section{Discussion}

In this study, in two large population-based cohorts of adults we found the presence of a restrictive spirometric pattern to be associated with respiratory symptoms and to affect significantly the physical, but not the mental, health component of quality of life. Of note, unlike participants with the obstructive pattern, those with the restrictive pattern had PCS deficits that remained significant after full adjustment for covariates, including respiratory symptoms and comorbidities.

Epidemiological studies have demonstrated conclusively that a restrictive spirometric pattern is present in a significant proportion of adults from the general population, and that it carries a substantial morbidity and mortality burden [2-4, 6-8, 18-24]. In addition to a notable geographic variability [6], the prevalence of the restrictive pattern is also highly dependent on the criteria used to define it (e.g. on whether spirometric cut-off values that are based on fixed levels, on \% predicted levels or on LLN thresholds are used). As our study included two cohorts that differed in their geographic and age distributions, we used definitions of spirometric patterns that were based on LLN values identified by predictive equations derived within each cohort. While this approach may have reduced the prevalence of abnormal spirometric patterns in our study, it minimises the potential impact of intercohort variability on the main 
TABLE 3 Associations between spirometry patterns and Physical Component Summary values in the follow-up of the European Community Respiratory Health Survey (ECRHS 2) and Swiss Cohort Study on Air Pollution and Lung and Heart Diseases in Adults (SAPALDIA 2)

\begin{tabular}{|c|c|c|c|c|}
\hline & \multicolumn{2}{|l|}{ ECRHS $\#$} & \multicolumn{2}{|l|}{ SAPALDIA ${ }^{\pi}$} \\
\hline & Coefficient $(95 \% \mathrm{CI})$ & $p>|z|$ & Coefficient $(95 \% \mathrm{CI})$ & $p>|z|$ \\
\hline \multicolumn{5}{|l|}{ M0: age+sex+education } \\
\hline Normal & 0 & & & 0 \\
\hline Restrictive pattern & $-3.07(-4.41--1.73)$ & $<0.001$ & $-2.73(-4.12--1.34)$ & $<0.001$ \\
\hline Obstructive pattern & $-1.73(-2.78--0.68)$ & 0.001 & $-1.55(-2.41--0.69)$ & $<0.001$ \\
\hline \multicolumn{5}{|l|}{ M1: M0+BMI categories } \\
\hline Normal & 0 & & & 0 \\
\hline Restrictive pattern & $-2.54(-3.88--1.21)$ & $<0.001$ & $-2.61(-3.98--1.23)$ & $<0.001$ \\
\hline Obstructive pattern & $-1.76(-2.81--0.72)$ & 0.001 & $-1.55(-2.39--0.70)$ & $<0.001$ \\
\hline \multicolumn{5}{|c|}{ M2: M1+smoking status and pack-years } \\
\hline Normal & 0 & & & 0 \\
\hline Restrictive pattern & $-2.36(-3.69--1.02)$ & 0.001 & $-2.59(-3.96--1.22)$ & $<0.001$ \\
\hline Obstructive pattern & $-1.59(-2.64--0.55)$ & 0.003 & $-1.28(-2.13--0.42)$ & 0.003 \\
\hline \multicolumn{5}{|l|}{ M3: M2+comorbidities ${ }^{+}$} \\
\hline Normal & 0 & & & 0 \\
\hline Restrictive pattern & $-2.11(-3.39--0.83)$ & 0.001 & $-2.51(-3.85--1.18)$ & $<0.001$ \\
\hline Obstructive pattern & $-1.32(-2.33--0.32)$ & 0.010 & $-1.21(-2.04--0.38)$ & 0.004 \\
\hline \multicolumn{5}{|c|}{ M4: M3+respiratory symptoms $\S$} \\
\hline Normal & 0 & & & 0 \\
\hline Restrictive pattern & $-1.89(-3.16--0.62)$ & 0.004 & $-2.13(-3.45--0.82)$ & 0.001 \\
\hline Obstructive pattern & $-0.49(-1.51-0.54)$ & 0.351 & $-0.62(-1.45-0.21)$ & 0.145 \\
\hline \multicolumn{5}{|c|}{$\begin{array}{l}\text { Coefficients are from linear mixed models after adjustment for various covariates. BMI: body mass index. } \\
{ }^{\#}: \mathrm{n}=4065 ;{ }^{\uparrow}: \mathrm{n}=4326 ;{ }^{+}: \text {comorbidities were used as an overall comorbidity score (see Materials and } \\
\text { methods), results were confirmed in models that included comorbidities as individual binary variables; } \\
\S_{\text {: }} \text { wheezing, chronic bronchitis, dyspnoea or asthma. }\end{array}$} \\
\hline
\end{tabular}

results. Notably, when using definitions of spirometric patterns that were based on fixed cut-offs and on \% predicted values, we observed substantial intercohort differences in the prevalence of spirometric patterns (possibly related to underlying intercohort differences in age, risk factors and procedures), but we could replicate associations of the restrictive spirometric pattern with PCS deficits in both ECRHS and SAPALDIA (supplementary table E2).

The nature and determinants of the restrictive spirometric pattern remain largely to be resolved [1]. In fact, in the absence of a direct measurement of total lung capacity, the extent to which the restrictive spirometric pattern actually identifies a true pulmonary restriction remains unknown. Several studies have suggested that spirometry has a good negative but a poor positive predictive value for true restriction, i.e. the vast majority of subjects with conserved vital capacity do not have pulmonary restriction, but only a proportion of subjects with spirometric restriction actually have low total lung capacity [25-27]. In addition, spirometric restriction may be due to air trapping associated with obstructive lung diseases and, in turn, it may represent a misclassified obstructive pattern. However, we argue that this scenario is unlikely to account for a substantial proportion of our cases of spirometric restriction for several reasons. First, longitudinal studies have demonstrated that only a minority of subjects with spirometric restriction develop an obstructive pattern over time [2]. Second, large-scale imagining studies have shown that smokers with spirometric restriction have distinct airway structural changes [28], but levels of air trapping and lung emphysema that are comparable to those of smokers with normal spirometry [5]. Finally, traditional risk factors for asthma and COPD show inconsistent associations with spirometric restriction.

In our study, the restrictive spirometric pattern was associated with significant deficits in the physical component of quality of life that were robust to statistical adjustment for demographic and behavioural factors, comorbidities, and respiratory symptoms (table 3). These results suggest that the restrictive pattern may influence quality of life through mechanisms that are at least partly independent of respiratory clinical impairment. This possibility is also supported by the observation that, when models were restricted to subjects who did not report any respiratory symptoms, the restrictive but not the obstructive group still had significant deficits in quality of life compared with normal subjects in both ECRHS and SAPALDIA. Interestingly, in a previous report from the ARIC (Atherosclerosis Risk in Communities) study, among participants with no chronic respiratory diagnoses or persistent respiratory symptoms, low FVC but not 
airway obstruction predicted poor survival, but results were substantially different when analyses included symptomatic subjects [29].

It is therefore possible that the restrictive pattern may include a diverse group of conditions and aetiologies that are not necessarily restricted to a primary involvement of the lung. Among them, at least for older subjects, there may be muscle weakness, sarcopenia and frailty. In this context, a recent report from the Cardiovascular Health Study found that, after adjustment for covariates, airflow limitation was associated with an $88 \%$ increase in the odds for being frail, but the restrictive pattern was associated with up to a $205 \%$ increase [30]. Alternatively, because the restrictive pattern has been consistently associated with diabetes, hypertension and cardiovascular disease, these diseases or factors that increase the risk for these diseases might also be involved in the aetiology of the spirometric pattern. Exposure to air pollution [31], low-grade systemic inflammation and obesity are some of these potential factors, although it should be pointed out that $<25 \%$ of participants with the restrictive pattern were obese in our study and that the association between spirometric restriction and PCS was confirmed after adjusting for obesity (table 3) and after removing obese participants (data not shown). Also, alterations of in utero and post-natal growth trajectories and developmental processes could impact multiple organs and, in turn, play a role in the achievement of maximal lung function levels in adulthood. Interestingly, we found ECRHS participants with a restrictive pattern to be more likely to report hospitalisation for lung disease in the first 2 years of life than those with normal lung function. Although this association is possibly susceptible to recall bias, it is noteworthy that early life events can play a critical role in lung function growth and tracking into adult life $[32,33]$, and their potential influence on the development of spirometric restriction in adult life warrants further investigation.

Among the limitations of our study is the possible presence of intercohort heterogeneity due to the different geographic and age distributions of ECRHS and SAPALDIA. However, it should be noted that, although prevalence rates of spirometric patterns defined by fixed cut-offs differed between the two cohorts, we used within-cohort sex- and age-specific LLN thresholds to minimise the potential impact of these differences. In addition, results of main analyses on quality of life were very consistent between the two cohorts using both the LLN and fixed cut-off definitions. We acknowledge that the SF-36 questionnaire may have missed specific respiratory components of quality of life and it was administered only at the follow-up survey, and that the assessment of comorbidities in our study may have been limited. Among the strengths of the study are the availability of two large, population-based epidemiological cohorts, the use of a standardised assessment of quality of life, the availability of extensive longitudinal lung function data, and highly consistent results on spirometric restriction and quality of life across the two cohorts.

\section{Conclusions}

In two large, population-based cohorts of adults we found that the association between the restrictive spirometric pattern and deficits in the physical component of quality of life was partly independent of the presence of respiratory symptoms. The identification of the factors and mechanisms underlying the restrictive pattern may contribute to effective strategies to improve the quality of life of these patients.

\section{Acknowledgements}

Author contributions: S. Guerra designed the study and drafted the manuscript with input from all authors. A-E. Carsin and D. Keidel analysed the data with input from all authors. All authors contributed to the interpretation of the data and approved the final version of the manuscript.

\section{References}

1 Godfrey MS, Jankowich MD. The vital capacity is vital: epidemiology and clinical significance of the restrictive spirometry pattern. Chest 2016; 149: 238-251.

2 Guerra S, Sherrill DL, Venker C, et al. Morbidity and mortality associated with the restrictive spirometric pattern: a longitudinal study. Thorax 2010; 65: 499-504.

3 Mannino DM, Ford ES, Redd SC. Obstructive and restrictive lung disease and functional limitation: data from the Third National Health and Nutrition Examination. J Intern Med 2003; 254: 540-547.

4 Soriano JB, Miravitlles M, Garcia-Rio F, et al. Spirometrically-defined restrictive ventilatory defect: population variability and individual determinants. Prim Care Respir J 2012; 21: 187-193.

5 Vaz Fragoso CA, McAvay G, Van Ness PH, et al. Phenotype of spirometric impairment in an aging population. Am J Respir Crit Care Med 2016; 193: 727-735.

6 Mannino DM, McBurnie MA, Tan W, et al. Restricted spirometry in the Burden of Lung Disease Study. Int $J$ Tuberc Lung Dis 2012; 16: 1405-1411.

7 Wheaton AG, Ford ES, Thompson WW, et al. Pulmonary function, chronic respiratory symptoms, and health-related quality of life among adults in the United States - National Health and Nutrition Examination Survey 2007-2010. BMC Public Health 2013; 13: 854.

8 Mannino DM, Buist AS, Petty TL, et al. Lung function and mortality in the United States: data from the First National Health and Nutrition Examination Survey follow up study. Thorax 2003; 58: 388-393.

9 Scarlata S, Pedone C, Fimognari FL, et al. Restrictive pulmonary dysfunction at spirometry and mortality in the elderly. Respir Med 2008; 102: 1349-1354. 
10 Leone N, Courbon D, Thomas F, et al. Lung function impairment and metabolic syndrome: the critical role of abdominal obesity. Am J Respir Crit Care Med 2009; 179: 509-516.

11 Ford ES, Mannino DM. Prospective association between lung function and the incidence of diabetes: findings from the National Health and Nutrition Examination Survey Epidemiologic Follow-up Study. Diabetes Care 2004; 27: 2966-2970.

12 Johnston AK, Mannino DM, Hagan GW, et al. Relationship between lung function impairment and incidence or recurrence of cardiovascular events in a middle-aged cohort. Thorax 2008; 63: 599-605.

13 Aaronson NK, Acquadro C, Alonso J, et al. International Quality of Life Assessment (IQOLA) Project. Qual Life Res 1992; 1: 349-351.

14 Ware JE, Kosinski M, Keller SD. SF-36 Physical and Mental Health Summary Scales: A User's Manual. Boston, The Health Institute, 1994.

15 Leynaert B, Neukirch C, Liard R, et al. Quality of life in allergic rhinitis and asthma. A population-based study of young adults. Am J Respir Crit Care Med 2000; 162: 1391-1396.

16 Svanes C, Sunyer J, Plana E, et al. Early life origins of chronic obstructive pulmonary disease. Thorax 2010; 65: $14-20$.

17 Hankinson JL, Odencrantz JR, Fedan KB. Spirometric reference values from a sample of the general U.S. population. Am J Respir Crit Care Med 1999; 159: 179-187.

18 Mannino DM, Doherty DE, Sonia Buist A. Global Initiative on Obstructive Lung Disease (GOLD) classification of lung disease and mortality: findings from the Atherosclerosis Risk in Communities (ARIC) study. Respir Med 2006; 100: 115-122.

19 Marcus BS, McAvay G, Gill TM, et al. Respiratory symptoms, spirometric respiratory impairment, and respiratory disease in middle-aged and older persons. J Am Geriatr Soc 2015; 63: 251-257.

20 Nonato NL, Nascimento OA, Padilla RP, et al. Occurrence of respiratory symptoms in persons with restrictive ventilatory impairment compared with persons with chronic obstructive pulmonary disease: the PLATINO study. Chron Respir Dis 2015; 12: 264-273.

21 Mannino DM, Thorn D, Swensen A, et al. Prevalence and outcomes of diabetes, hypertension and cardiovascular disease in COPD. Eur Respir J 2008; 32: 962-969.

22 Mannino DM, Holguin F, Pavlin BI, et al. Risk factors for prevalence of and mortality related to restriction on spirometry: findings from the First National Health and Nutrition Examination Survey and follow-up. Int $J$ Tuberc Lung Dis 2005; 9: 613-621.

23 Scarlata S, Costanzo L, Giua R, et al. Diagnosis and prognostic value of restrictive ventilatory disorders in the elderly: a systematic review of the literature. Exp Gerontol 2012; 47: 281-289.

24 Fimognari FL, Pasqualetti P, Moro L, et al. The association between metabolic syndrome and restrictive ventilatory dysfunction in older persons. J Gerontol A Biol Sci Med Sci 2007; 62: 760-765.

25 Aaron SD, Dales RE, Cardinal P. How accurate is spirometry at predicting restrictive pulmonary impairment? Chest 1999; 115: 869-873.

26 Swanney MP, Beckert LE, Frampton CM, et al. Validity of the American Thoracic Society and other spirometric algorithms using FVC and forced expiratory volume at $6 \mathrm{~s}$ for predicting a reduced total lung capacity. Chest 2004; 126: $1861-1866$.

27 Vandevoorde J, Verbanck S, Schuermans D, et al. Forced vital capacity and forced expiratory volume in six seconds as predictors of reduced total lung capacity. Eur Respir J 2008; 31: 391-395.

28 Wan ES, Hokanson JE, Murphy JR, et al. Clinical and radiographic predictors of GOLD-unclassified smokers in the COPDGene study. Am J Respir Crit Care Med 2011; 184: 57-63.

29 Burney PG, Hooper R. Forced vital capacity, airway obstruction and survival in a general population sample from the USA. Thorax 2011; 66: 49-54.

30 Vaz Fragoso CA, Enright PL, McAvay G, et al. Frailty and respiratory impairment in older persons. Am J Med 2012; 125: 79-86.

31 de Jong K, Vonk JM, Zijlema WL, et al. Air pollution exposure is associated with restrictive ventilatory patterns. Eur Respir J 2016; 48: 1221-1224.

32 Stocks J, Hislop A, Sonnappa S. Early lung development: lifelong effect on respiratory health and disease. Lancet Respir Med 2013; 1: 728-742.

33 Stern DA, Morgan WJ, Wright AL, et al. Poor airway function in early infancy and lung function by age 22 years: a non-selective longitudinal cohort study. Lancet 2007; 370: 758-764. 